\title{
Exopolysaccharides produced by Lactobacillus plantarum: technological properties, biological activity, and potential application in the food industry
}

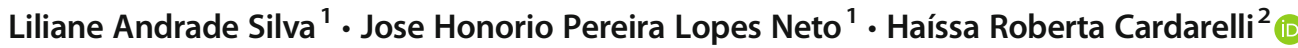

Received: 26 November 2018 / Accepted: 21 February 2019 /Published online: 2 March 2019

(C) Università degli studi di Milano 2019

\begin{abstract}
Some lactic acid bacteria are capable of producing capsular or extracellular polysaccharides, with desirable technological properties and biological activities. Such polysaccharides produced by lactic acid bacteria are called exopolysaccharides and can be used to alter rheological properties, acting in processes involving viscosity, emulsification, and flocculation, among others. They may also be involved in prebiotic, probiotic, and biological activities, as well as having potential application in the food industry. In this mini-review, the objectives were to present some beneficial properties of exopolysaccharides (EPS) produced by Lactobacillus plantarum that have not been commercially explored. For that, the article focused to summarize revision of current publications within the following topics: (1) rheological properties, (2) prebiotic properties, (3) biological activities, and (4) potential application in the food industry. EPS produced by Lb. plantarum can be used as gelling agent, emulsifier, or stabilizer for food products. The glucan nature of the produced EPS enhances probiotic properties of this LAB species. Lactobacillus plantarum EPS has antioxidant, antibiofilm, and antitumor activities. Finally, there is an improvement in texture of fermented food products where $L b$. plantarum is used as starter culture which is related to EPS production in situ. Therefore, EPS produced by $L$. plantarum have important and desirable properties to be explored for several applications, including health and food areas.
\end{abstract}

Keywords EPS $\cdot$ Lactic acid bacteria $\cdot$ Potential activity $\cdot$ Beneficial properties

\section{Introduction}

Some species of lactic bacteria are able to synthesize and excrete extracellular polysaccharides, called exopolysaccharides (EPS). The production of exopolysaccharides (EPS) by lactic acid bacteria (LAB) has gained special interest over the last decade due to functional properties of these bio-polymers as well as their generally recognized as safe (GRAS) status (Dertli et al. 2013, 2016). Under specific growth conditions, Lactobacillus species generate a wide range and diversity of

Haíssa Roberta Cardarelli hrcarda@gmail.com

1 Postgraduate Program in Food Science and Technology, Department of Food Engineering, Federal University of Paraíba (UFPB), Cidade Universitária, João Pessoa, PB 58051-900, Brazil

2 Department of Food Technology, Center for Technology and Regional Development, Federal University of Paraíba, João Pessoa, Brazil
EPS structures that can be used as potential application in the food and pharmaceutical industry (Donot et al. 2012; Zannini et al. 2016).

EPS are divided by their structure into two groups: heteropolysaccharides and homopolysaccharides. The synthesis of homopolysaccharides (HoPS) occurs in the extracellular environment from the disaccharide sucrose, which acts in the granting of monosaccharides (fructose or glucose), from the enzymatic digestion inserted in the family of glycosyl hydrolase (GH) (Van Hijum et al. 2004, 2006; Leemhuis et al. 2013; Zannini et al. 2016). In contrast to HoPS, heteropolysaccharides (HePS) require in their formation the intracellular scope, containing repeating units, and, in addition, lipids such as isoprenoid glycosyl may participate in the development. However, the polymerization occurs outside the cell, preceded by the displacement of the repeating units via the membrane. Fermentation conditions define the amount and type of EPS produced, as well as, biosynthesis and secretion of microbial EPS are dependent on the various phases of microorganism growth (De Vuyst and Degeest 1999; Zannini et al. 2016). 
EPS provide some important rheological characteristics such as texture and body of certain foodstuffs. These physical properties are not only influenced by the amount of biopolymer but also its structure, considering size and frequency of branching and molecular mass (Duboc and Mollet 2001; Ruas-Madiedo et al. 2002), in addition to the interactions between polysaccharides (De Vuyst and Degeest 1999). EPS are also known to display several health-promoting effects such as anti-tumor, antioxidant, immune modulating, and prebiotic and probiotic properties and hence are considered valuable ingredients in the food industry (Das et al. 2014). Lactobacillus plantarum has been reported as a producer of exopolysaccharides with various properties and activities essential for commercialization by the food, cosmetic, or pharmaceutical industries (Das et al. 2014; Fontana et al. 2015; Oh and Jung 2015; Zhang et al. 2016). This study aimed to report a short review of the main biological properties and activities presented by $L b$. plantarum.

Figure 1 compiles the main functions of L. plantarum EPS which will be detailed along this review.

\section{Composition of Lactobacillus plantarum EPS}

The analytical methodologies for EPS composition produced by $L b$. plantarum have been described as hydrolysis and derivatization of the resultant monosaccharides, followed by chromatographic methods (Zhang et al. 2016).
There are many reports in the literature on $L b$. plantarum-produced exopolysaccharides containing portions of galactose, glucose, and mannose. Monosaccharide compositional analysis by HPLC has revealed the presence of independent peaks in Lb. plantarum NTMI05 and NTMI20, confirming the presence of galactose (Imran et al. 2016). Compositional analysis of $L b$. plantarum YW32 EPS by GC-MS has shown that it is composed of different sugar monomers such as mannose, fructose, galactose, and glucose, suggesting that it is a heteropolysaccharide. In fact, several $\mathrm{Lb}$. plantarum strains have also been previously shown to produce different heteropolysaccharides. For example, EPS produced by $L b$. plantarum NTU 102 containing fructose, arabinose, galactose, glucose, mannose, and maltose in a different polarity ratio, and three monomers such as mannose, glucose, and galactose were found in the EPS produced by $L b$. plantarum KF5 (Wang et al. 2010; 2015a, b; Liu et al. 2011).

Carbohydrates represent about 90 to $96 \%$ of the EPS composition produced by $L b$. plantarum (Wang et al. 2014; Imran et al. 2016). Other components may be related in their composition as well, but at very low levels, for instance, sulfated groups, protein, and nucleic acids as well as uronic acid (Wang et al. 2014; Zhang et al. 2016).

\section{Rheological properties}

Among the rheological properties of EPS, the solubility, water holding capacity, emulsification, viscosity, and flocculation
Fig. 1 Functions of $L b$. plantarum EPS

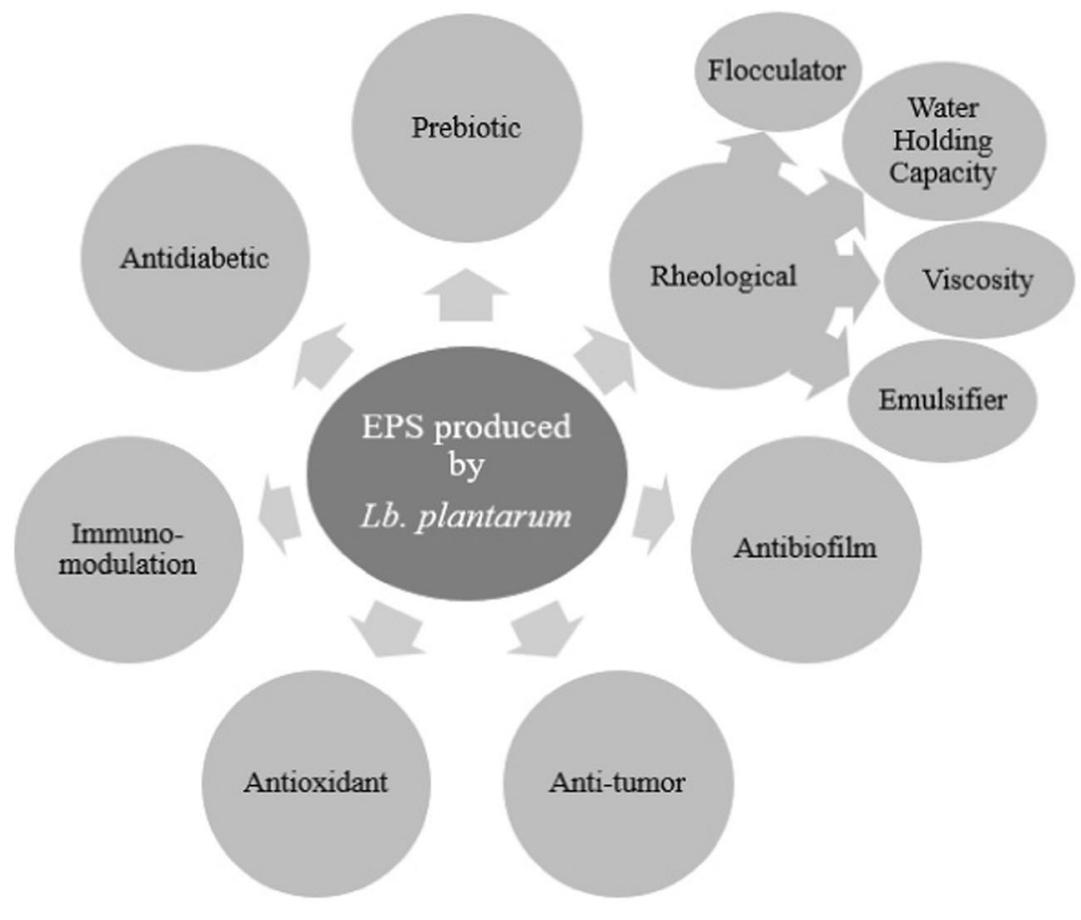


properties are the most used for industrial application of these polysaccharides. Solubility and water retention capacity are properties associated with the porous matrix structure formed by polysaccharide chains which may contain large amounts of water via hydrogen bonds, as already reported by Zhu et al. (2014). The good solubility of some EPS depends to a large extent on their molecular weight and the percentage of branching (Tsusaki et al. 2009). Due to their good solubility and water retention capacity, some EPS such as D-glucan DM5 can be used as emulsifiers or stabilizers for food products (Das et al. 2014). The same authors reported $L b$. plantarum DM5 produced D-glucan containing $86.5 \%$ $\alpha-(1 \rightarrow 6)$ and $13.5 \%$ branched linkages $\alpha-(1 \rightarrow 3)$ and exhibited a non-toxic biocompatible nature with unique rheological properties, thus facilitating its potential use as a gelling agent in the food industry.

Some EPS have shown a trend of flocculation activity (Wang et al. 2008), which is interesting because they are considered natural flocculants and biodegradable and potential substitutes for chemical-synthesized flocculants (Salehizadeh and Shojaosadati 2001; Zhang et al. 2007a, b) and may be used together with other natural flocculants, e.g., chitosan frequently used (Salehizadeh et al. 2000). Viscosity is another crucial property to produce the desired attributes. Lactic acid bacteria addition into foods has been proposed to increase viscosity, given its ability to produce biopolymers in situ (Ryan et al. 2015). Russo et al. (2016) reported that Lb. plantarum strains were used for oat fermentation, and produced EPS Lp90 that favored rheological characteristics of the product, although it was apparently lost during storage.

The textural and microstructural impact of EPS produced by $L b$. plantarum isolated from camel milk on low-fat akawi cheese was also reported (Ayyash et al. 2018). Cheeses containing two different EPS positively altered the parameters of hardness and chewiness after 7 days of refrigerated storage, with smaller pores in the microstructure, and modified water retention capacity, which influenced the rheologic parameters.

Thus, it can be seen that the EPS produced by $L b$. plantarum have been largely studied recently (Das et al. 2014; Oh and Jung 2015; Wang et al. 2015a, b; Russo et al. 2016), proving their potential application given their demonstrated rheological properties.

\section{Prebiotic properties}

Strains of lactic acid bacteria (LAB) and EPS producers including $L b$. plantarum have been reported as having prebiotic and probiotic effects. Concerning prebiotic action, it was noticed that EPS could be used by certain probiotic strains which possess the enzymes necessary to degrade EPS (Tsuda and
Miyamoto 2010). Prebiotic activity is "the ability of a given substrate to support the growth of an organism relative to other organisms and relative to growth on a non-prebiotic substrate, such as glucose" (Huebner et al. 2007). Das et al. (2014) demonstrated stimulation on the growth of Lb. plantarum DM5 in the presence of glucan-DM5, which is produced by the strain and inulin, achieving higher prebiotic scores. Conversely, non-probiotic strains such as Escherichia coli and E. aerogenes did not succeed in growing. Moreover, EPS from $L b$. plantarum demonstrated significant resistance to hydrolysis by gastric juice and intestinal digestion. Regarding all the abovementioned benefits, EPS produced by $L b$. plantarum could be considered as having potential prebiotic effects.

Probiotic survival can be influenced by the EPS produced by lactic acid bacteria (LAB) during the gastrointestinal transit (Caggianiello et al. 2016). Some studies have affirmed to have enhanced the growth and stress tolerance of probiotic Lactobacilli with the addition of microbial polysaccharides such as glucans. However, positive effects of the presence of EPS on some Lactobacillus spp. strains survival have not been noticed in the human digestive tract. This might be due to the compositions and particular chemical structures of the tested microbial EPS, which are related to strain specificity and ability to produce different EPS and its capacity to interact with the host epithelium in the intestinal tract, including bacterial surface properties as well as survival under the stress conditions found in the gastrointestinal tract (acid and bile stress) (Looijesteijn et al. 2001; De Palencia et al. 2009; Russo et al. 2012; Caggianiello et al. 2016; Lee et al. 2016).

Bacterial probiotic potential is related to its effectiveness against harsh gastrointestinal conditions through adhering to the gut mucosa, fighting pathogens by production of antimicrobial compounds, and/or provoking beneficial effects on human health (Servin 2004). Moreover, bacteria should be resistant to bile, low $\mathrm{pH}$, and antibiotics and possess antimicrobial activity (Kruszewska et al. 2002; Lee and Salminen 2009). Likewise, Lb. plantarum has proved to have some of the before mentioned probiotic properties such as good acid tolerance, survival at bile concentration $(0.3-1 \%)$, adhesion properties, good cholesterol removal rates $(58 \%)$, the highest sodium nitrite depletion rates, and antibacterial and exceptional antioxidative activities, in addition to considerable EPS production by $L b$. plantarum (1557) isolated from vegetables (Ren et al. 2014). It has already been stated that probiotic survival could depend on the EPS produced by the probiotic strain, and therefore $L b$. plantarum has this combined ability also associated to EPS production to be converted into probiotic and prebiotic ingredients. Recently, the functional properties of exopolysaccharide produced by $L b$. plantarum CIDCA 8327 isolated from Kefir was reported (Gangoiti et al. 2017) and indicated as being promising for the development of functional foods. 


\section{Biological activity}

\section{Antioxidant activity}

EPS obtained from the cell-free supernatant of LAB exhibit antioxidant activities (Kodali and Sen 2008; Liu et al. 2011; Oh and Jung 2015). EPS produced by Lb. plantarum C88 was confirmed to be involved in the antioxidant activity of this strain since the purified EPS exhibited strong in vitro radical scavenging activity and antioxidant activity against $\mathrm{H}_{2} \mathrm{O}_{2}$-induced injury in Caco-2 cells (Zhang et al. 2013; Fontana et al. 2015).

Imran et al. (2016) used different reaction mechanisms to evaluate EPS antioxidant activities, including DPPH free radical scavenging activity and reducing power assay. The authors reported the highest scavenging activity of EPS was observed at a $500 \mu \mathrm{g} / \mathrm{mL}$ concentration of the EPS from $\mathrm{Lb}$. plantarum NTMI05 and NTMI20 with $96.62 \%$ and $91.86 \%$, respectively. The antioxidant activity may be due to the presence of hydroxyl group and other functional groups in EPS of both strains which can donate electrons to reduce the radicals to a more stable form, or to react with the free radicals to terminate the radical chain reaction (Shen et al. 2013). Dilna et al. (2015) also reported that 2 (two) $\mathrm{mg} / \mathrm{mL}$ of $L b$. plantarum RJF4 EPS exhibited $23.63 \%$ DPPH radical removal activity. Wang et al. (2014) evaluated antioxidant properties of r-EPS1 and r-EPS2 produced by $L b$. plantarum 70810. In general, r-EPS2 presented higher antioxidant activities than r-EPS1, which was probably attributed to the presence of sulfated groups in r-EPS2. Hydroxyl scavenging activities were $69.81 \%$ for EPS2 at the highest concentration $(4.0 \mathrm{mg} / \mathrm{mL})$, while r-EPS1 and r-EPS2 $(4.0 \mathrm{mg} / \mathrm{ml})$ also showed good radical DPPH scavenging activities (27.98\% and $48.43 \%$, respectively). These results imply that r-EPS of $L b$. plantarum 70810 may be good hydrogen or electron donors. In addition, absorbances of reducing power test were 0.359 and 0.423 for-EPS1 and r-EPS2 $(4.0 \mathrm{mg} / \mathrm{mL})$, respectively. These results demonstrate there might be a correlation between radical scavenging activity and reducing power.

\section{Anti-tumor activity}

Studies report that the composition of monosaccharides and glycosidic linkage plays a role in the anti-tumor activities of monosaccharides (Zhang et al. 2007a, b). EPS from LAB have been found to have significant antioxidant and anti-tumor activities, and they have drawn attention recently. In assays performed by Wang et al. (2014), the inhibitory effects of the purified fractions of r-EPS (secreted into the surrounding medium as released exopolysaccharides) produced by $L b$. plantarum added to Caco-2 human colon carcinin, BGC-823 gastric cancer, and colon cancer HT-29 cells in vitro were evaluated using the Tetrazoliun dye uptake assay (mitochondrial metabolic activity) (MTT) in 96-well plates. The results revealed inhibition ratios of r-EPS1 and r-EPS2 at different concentrations for $24 \mathrm{~h}$ against
Caco-2, BGC-823, and HT-29 cells. Both r-EPS1 and r-EPS2 exhibited inhibitory effects on the proliferation of these tumor cells, and inhibition activities increased along with increasing concentrations of exopolysaccharides. At the highest concentration $(600 \mu \mathrm{g} / \mathrm{mL})$, the inhibition ratios against Caco-2 were $25.94 \pm 1.98 \%$ and $35.04 \pm 0.87 \%$ for r-EPS1 and r-EPS2, respectively. Apparently, r-EPS2 had higher inhibitory effects on growth in the investigated tumor cells than r-EPS1. This is justified by the presence of sulfated group and beta-glycosidic bond composition in r-EPS2.

Liu et al. (2009) also reported that sulfated polysaccharides had higher anti-tumor activity than non-sulfated polysaccharides. In the study of anti-tumor activity performed by Wang et al. (2015b), EPS produced by Lb. plantarum YW32 exerted inhibitory activities on HT-29 cells in concentration dependence modes. After $72 \mathrm{~h}$ of treatment, a stronger inhibitory rate (39.24\%) of EPS was observed at a concentration of $600 \mu \mathrm{g} / \mathrm{ml}$. The anti-tumor activity of $L b$. plantarum YW32 EPS was related to its relatively strong scavenging abilities in relation to hydroxyl and superoxide radicals, suggesting that the Lb. plantarum YW32 EPS may be a potentially natural food for colon cancer therapy. Anti-proliferative activities on HT-29 cells have also been reported with other EPS from L. acidophilus (Kim et al. 2010) and L. casei (Liu et al. 2011). Differences in the anti-tumor activities of these EPS may be related to their different phytochemical characteristics, including their specific conformation, expanded chain, molecular weight, and composition of monosaccharides (Wu et al. 2014).

\section{Antibiofilm activity}

Some bacterial pathogens demonstrate the ability to form biofilms, causing food spoilage. Some Lactobacillus have been described as having antimicrobial activity against several biofilm-forming bacteria (Zhang et al. 2013; Li et al. 2014; Wang et al. 2015b). EPS may mediate antimicrobial activity by modifying the surfaces of bacterial cells by inhibiting the initial binding of bacterial cells to the surface, or by acting as signaling molecules that regulate the gene expression involved in biofilm formation (Kim and Kim 2009; Rendueles et al. 2013).

Lactobacillus plantarum YW32 EPS was tested in the formation of biofilms of four pathogens: Escherichia coli $\mathrm{O} 157$, Shigella flexneri CMCC (B), Staphylococcus aureus AC1, and Salmonella typhimurium S50333, where an increase in biofilm inhibition as the concentration of EPS increased from 0.2 to $5.0 \mathrm{mg} / \mathrm{ml}$ was observed. EPS showed higher inhibition of Staphylococcus aureus AC1 (45.13\%), Shigella flexneri CMCC (B) (44.67\%), and Salmonella typhimurium S50333 (44.04\%) biofilm formation when compared to Escherichia coli $\mathrm{O} 157$ (only $12.71 \%$ ). These results indicate that EPS of Lb. plantarum YW32 has a broad spectrum of antibiofilm activity, which would be advantageous for application in the food industry as food grade adjuvants to control microbial 
biofilm formation. Bacterial EPS, including Lb. plantarum YW32 EPS, had the ability to repress the biofilm formed by both Gram-negative and Gram-positive pathogens. However, the molecular mechanism mediated the antibiofilm activity of the $L b$. plantarum YW32 EPS does not appear to be due to the bactericidal effect since none of the EPS had biocidal activity (Wang et al. 2015b).

Chart 1 compiles information about EPS producers Lb.plantarum strains, substrate of isolation, its biological activity, and technological properties.

\section{Potential application of EPS in the food industry}

The industrial applications of microbial exopolysaccharides are a topic of growing interest that have been extensively reviewed by Zannini et al. (2016). There is a wide range of EPS-producing bacteria with interesting industrial applications, such as xanthan, gellan (Donot et al. 2012), dextran, and pullulan (Lopez et al. 2005). EPS from LAB, mainly heteropolysaccharides (HePS), have been widely used in the dairy industry, and mainly when they are produced in situ during fermentation to improve emulsifying and thickening properties for syneresis reduction and firmness increase (Zisu and Shah 2005; Dabour et al. 2006; Ale et al. 2016). The availability of LAB starter cultures which produce exopolysaccharides in situ during fermentation could be a suitable alternative for products whose polysaccharide addition requires a specification as food additives, which is a condition that is not very much appreciated by consumers (Lahtinen et al. 2011). There is also increasing evidence that EPS exert a positive impact on human health. The low yield of HePS generally prevents its commercial use, in contrast to homopolysaccharides (HoPS) such as dextran from other microbial sources (Fanning et al. 2012; Ale et al. 2016). Currently, the highest production of microbial exopolysaccharides is attributed to Xanthomonas campestris, which produces $30-50 \mathrm{~g} / \mathrm{L}$ of xanthan gum, an extracellular heteropolysaccharide used as a food additive and rheology modifier, and whose industrial use is considered convenient. Although the EPS yield produced by LAB is much lower, the in situ applications in the manufacturing sector may be sustainable (Harutoshi 2013).

Lactobacillus plantarum (Ismail and Nampoothiri 2010), Lb. plantarum KF5 (Wang et al. 2010), and Lb. plantarum MTCC 9510 (Ismail and Nampoothiri 2014) are cited as promising producers of exopolysaccharides for the food industry. The main characteristics are related to an improvement in the texture of products, with the last EPS reported as having nonNewtonian pseudoplastic behavior and avoiding syneresis in products. However, it should be ensured that EPS do not pose any risk in order to be applied in the food industry. Sasikumar et al. (2017) investigated the cellular cytotoxicity of EPS produced by $L b$. plantarum $\mathrm{BR} 2$ and the results revealed that EPS is non-toxic to normal cells. From this result, the author reports that the studied EPS can be very useful for the application in the food industry in order to provide products with defined consistency and for the purpose of health promotion (functional).

Lactobacillus plantarum KX041 (Wang et al. 2017) is a promisingly microorganism-producing exopolysaccharide served as natural alternative for commercial additives. Moreover, the use of EPS produced by Lb. plantarum YW11 was reported by Wang et al. (2015b) as technically viable because the substance showed higher viscosity in skim milk and $\mathrm{pH}$ around 4; both parameters are favorable for addition in dairy products. Ayyash et al. (2018) showed a promising production of EPS Lb. plantarum isolated from camel milk, and that the strain exhibited comparable EPS production capabilities for a commercial crop of EPS production, in addition to being used as a starter culture for cheese production.

According to Freitas et al. (2017), the industrial development of microbial EPS processes and commercialization of the biopolymers require better understanding of their structural configurations and physico-chemical properties, which is scarce or unavailable for many of the reported polymers. The fermentation stage of most EPS production processes is still poorly monitored in real time, and/or poorly integrated with the unit operations downstream. Many microbial EPS hold great potential for development due to their novel and distinct properties compared to polysaccharides obtained from other natural sources. However, in order for their industrialization and commercialization to become attractive, it is necessary to put more effort not only into the fermentation stage to increase the productivity and lower production costs, but mainly into polymer characterization and the proof-of-concept of their application in high-value pharmaceutical, food, and cosmetic areas, where product quality and functional properties are far more relevant than production cost. Hence, the greatest potential for the development of novel microbial EPS is in those high-value market niches.

\section{Conclusion}

EPS produced by $L b$. plantarum have several important and desirable properties. Some of these properties still require more in-depth studies aimed at strain specific $L b$. plantarum, particularly when it comes to their possible industrial application in foods. The rheological and prebiotic properties are the main characteristics demonstrated by EPS produced by this microorganism. However, based on what has been reported in the most recent research and what has been cited in this study, there are very promising EPS with several applications, including health regarding antitumor and antioxidant activities and prebiotic effects. 


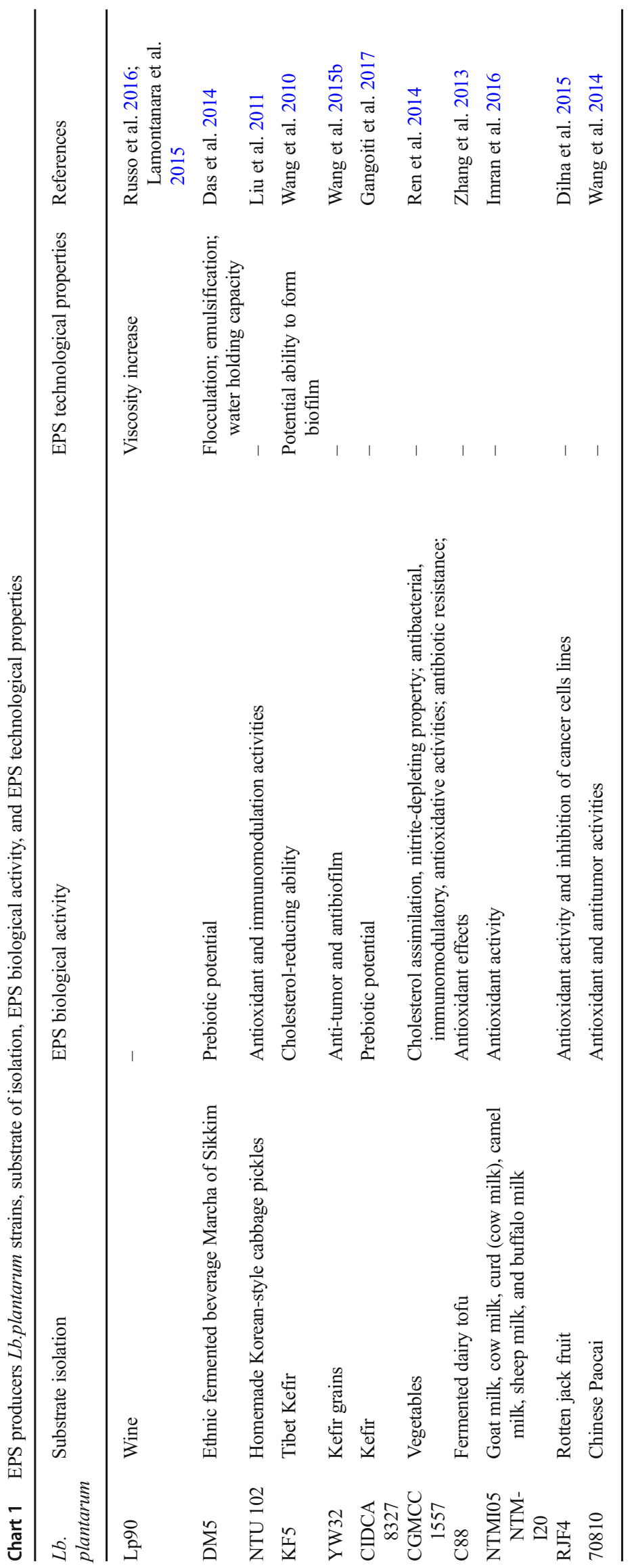


Acknowledgments The authors gratefully acknowledge the Brazilian Federal Agency for the Support and Evaluation of Graduate Education (Coordenação de Aperfeiçoamento de Pessoal de Nível Superior CAPES) for the granted fellowships.

Funding This study received research grants from funding agencies (Brazilian Federal Agency for the Support and Evaluation of Graduate Education (Coordenação de Aperfeiçoamento de Pessoal de Nível Superior - CAPES).

\section{Compliance with ethical standards}

Conflict of interest The authors declare that they have no conflicts of interest.

Research involving human participants and/or animals This article does not contain any studies with human participants or animals performed by any of the authors.

Informed consent Informed consent was obtained from all individual participants included in the study.

Publisher's note Springer Nature remains neutral with regard to jurisdictional claims in published maps and institutional affiliations.

\section{References}

Ale EC, Perezlindo MJ, Pavón Y, Peralta GH, Costa S, Sabbag N, Bergamini C, Reinheimer JA, Binetti AG (2016) Technological, rheological and sensory characterizations of a yogurt containing an exopolysaccharide extract from Lactobacillus fermentum Lf2, a new food additive. Food Res Int 90:259-267

Ayyash M, Abu-Jdayil B, Hamed F, Shaker R (2018) Rheological, textural, microstructural and sensory impact of exopolysaccharideproducing Lactobacillus plantarum isolated from camel milk on low-fat akawi cheese. LWT Food Sci Technol 87:423-431

Caggianiello G, Kleerebezem M, Spano G (2016) Exopolysaccharides produced by lactic acid bacteria: from health-promoting benefits to stress tolerance mechanisms. Appl Microbiol Biotechnol 100(9): 3877-3886

Dabour N, Kheadr E, Benhamou N, Fliss I, LaPointe G (2006) Improvement of texture and structure of reduced-fat Cheddar cheese by exopolysaccharide-producing lactococci. J Dairy Sci 89(1):95-110

Das D, Baruah R, Goyal A (2014) A food additive with prebiotic properties of an $\alpha$-d-glucan from Lactobacillus plantarum DM5. Int J Biol Macromol 69:20-26

De Palencia PF, Werning ML, Sierra-Filardi E, Dueñas MT, Irastorza A, Corbí AL, López P (2009) Probiotic properties of the 2-substituted (1, 3)- $\beta$-D-glucan-producing bacterium Pediococcus parvulus 2.6. Appl Environ Microbiol 75(14):4887-4891

De Vuyst L, Degeest B (1999) Heteropolysaccharides from lactic acid bacteria. FEMS Microbiol Rev 23(2):153-177

Dertli E, Colquhoun IJ, Gunning AP, Bongaerts RJ, Le Gall G, Bonev BB, Mayer MJ, Narbad A (2013) Structure and biosynthesis of two exopolysaccharides produced by Lactobacillus johnsonii FI9785. J Biol Chem 288(44):31938-31951

Dertli E, Toker OS, Durak MZ, Yilmaz MT, Tatlısu NB, Sagdic O, Cankurt H (2016) Development of a fermented ice-cream as influenced by in situ exopolysaccharide production: rheological, molecular, microstructural and sensory characterization. Carbohydr Polym $136: 427-440$
Dilna SV, Surya H, Aswathy RG, Varsha KK, Sakthikumar DN, Pandey A, Nampoothiri KM (2015) Characterization of an exopolysaccharide with potential health-benefit properties from a probiotic Lactobacillus plantarum RJF 4. LWT Food Sci Technol 64(2):1179-1186

Donot F, Fontana A, Baccou JC, Schorr-Galindo S (2012) Microbial exopolysaccharides: main examples of synthesis, excretion, genetics and extraction. Carbohydr Polym 87(2):951-962

Duboc P, Mollet B (2001) Applications of exopolysaccharides in the dairy industry. Int Dairy J 11(9):759-768

Fanning S, Hall LJ, Cronin M, Zomer A, MacSharry J, Goulding D, Motherway MO, Shanahan F, Nally K, Dougan G, Sinderen D (2012) Bifidobacterial surface-exopolysaccharide facilitates commensal-host interaction through immune modulation and pathogen protection. Proc Natl Acad Sci 109(6):2108-2113

Fontana C, Li S, Yang Z, Widmalm G (2015) Structural studies of the exopolysaccharide from Lactobacillus plantarum C88 using NMR spectroscopy and the program CASPER. Carbohydr Res 402:87-94

Freitas F, Torres CA, Reis MA (2017) Engineering aspects of microbial exopolysaccharide production. Bioresour Technol 245:1674-1683

Gangoiti MV, Puertas AI, Hamet MF, Peruzzo PJ, Llamas MG, Medrano M, Pietro A, Dueñas MT, Abraham AG (2017) Lactobacillus plantarum CIDCA 8327: an $\alpha$-glucan producing-strain isolated from kefir grains. Carbohydr Polym 170:52-59

Harutoshi T (2013) Exopolysaccharides of lactic acid bacteria for food and colon health applications. In: Kongo M (ed) Lactic acid bacteria-R \& D for food, health and livestock purposes. InTech, Tokyo. https://doi.org/10.5772/50839. Available from: https:// www.intechopen.com/books/lactic-acidbacteria-r-d-for-foodhealth-and-livestock-purposes/exopolysaccharides-of-lactic-acidbacteria-for-foodand-colon-health-applications

Huebner J, Wehling RL, Hutkins RW (2007) Functional activity of commercial prebiotics. Int Dairy J 17(7):770-775

Imran MYM, Reehana N, Jayaraj KA, Ahamed AAP, Dhanasekaran D, Thajuddin N, Alharbi NS, Muralitharan G (2016) Statistical optimization of exopolysaccharide production by Lactobacillus plantarum NTMI05 and NTMI20. Int J Biol Macromol 93:731-745

Ismail B, Nampoothiri KM (2010) Production, purification and structural characterization of an exopolysaccharide produced by a probiotic Lactobacillus plantarum MTCC 9510. Arch Microbiol 192(12): 1049-1057

Ismail B, Nampoothiri KM (2014) Molecular characterization of an exopolysaccharide from a probiotic Lactobacillus plantarum MTCC 9510 and its efficacy to improve the texture of starchy food. J Food Sci Technol 51(12):4012-4018

Kim Y, Kim SH (2009) Released exopolysaccharide (r-EPS) produced from probiotic bacteria reduce biofilm formation of enterohemorrhagic Escherichia coli O157: H7. Biochem Biophys Res Commun 379(2):324-329

Kim Y, Oh S, Yun HS, Kim SH (2010) Cell-bound exopolysaccharide from probiotic bacteria induces autophagic cell death of tumour cells. Lett Appl Microbiol 51(2):123-130

Kodali VP, Sen R (2008) Antioxidant and free radical scavenging activities of an exopolysaccharide from a probiotic bacterium. Biotechnol J 3(2):245-251

Kruszewska D, Lan J, Lorca G, Yanagisawa N, Marklinder I, Ljungh A (2002) Selection of lactic acid bacteria as probiotic strains by in vitro tests. Microb Ecol Health Dis 29:37-49

Lahtinen S, Ouwehand AC, Salminen S, von Wright A (eds) (2011) Lactic acid bacteria: microbiological and functional aspects. CRC Press, New York

Lamontanara A, Caggianiello G, Orrù L, Capozzi V, Michelotti V, Bayjanov JR, Renckens B, Van Hijum SAFT, Luigi C, Spano G (2015) Draft genome sequence of Lactobacillus plantarum Lp90 isolated from wine. Genome Announc 3(2):e00097-e00015 
Lee YK, Salminen S (2009) Handbook of probiotics and prebiotics. John Wiley \& Sons, New Jersey

Lee IC, Caggianiello G, van Swam II, Taverne N, Meijerink M, Bron PA, Spano G, Kleerebezem M (2016) Strain-specific features of extracellular polysaccharides and their impact on Lactobacillus plantarum-host interactions. Appl Environ Microbiol 82(13): 3959-3970

Leemhuis H, Pijning T, Dobruchowska JM, Van Leeuwen SS, Kralj S, Dijkstra BW, Dijkhuizen L (2013) Glucansucrases: threedimensional structures, reactions, mechanism, $\alpha$-glucan analysis and their implications in biotechnology and food applications. J Biotechnol 163(2):250-272

Li W, Ji J, Rui X, Yu J, Tang W, Chen X, Jiang M, Dong M (2014) Production of exopolysaccharides by Lactobacillus helveticus MB2-1 and its functional characteristics in vitro. LWT Food Sci Technol 59(2):732-739

Liu J, Luo J, Ye H, Sun Y, Lu Z, Zeng X (2009) Production, characterization and antioxidant activities in vitro of exopolysaccharides from endophytic bacterium Paenibacillus polymyxa EJS-3. Carbohydr Polym 78(2):275-281

Liu CF, Tseng KC, Chiang SS, Lee BH, Hsu WH, Pan TM (2011) Immunomodulatory and antioxidant potential of Lactobacillus exopolysaccharides. J Sci Food Agric 91(12):2284-2291

Looijesteijn PJ, Trapet L, de Vries E, Abee T, Hugenholtz J (2001) Physiological function of exopolysaccharides produced by Lactococcus lactis. Int J Food Microbiol 64(1):71-80

Lopez EC, Champion D, Blond G, Meste ML (2005) Influence of dextran, pullulan and gum arabic on the physical properties of frozen sucrose solutions. Carbohydr Polym 59(1):83-91

Oh YJ, Jung DS (2015) Evaluation of probiotic properties of Lactobacillus and Pediococcus strains isolated from Omegisool, a traditionally fermented millet alcoholic beverage in Korea. LWT Food Sci Technol 63(1):437-444

Ren D, Li C, Qin Y, Yin R, Du S, Ye F, Liu C, Liu H, Wang M, Li Y, Sun Y, Li X, Tian M, Jin N (2014) In vitro evaluation of the probiotic and functional potential of Lactobacillus strains isolated from fermented food and human intestine. Anaerobe 30:1-10

Rendueles O, Kaplan JB, Ghigo JM (2013) Antibiofilm polysaccharides. Environ Microbiol 15(2):334-346

Ruas-Madiedo P, Hugenholtz J, Zoon P (2002) An overview of the functionality of exopolysaccharides produced by lactic acid bacteria. Int Dairy J 12(2):163-171

Russo P, López P, Capozzi V, De Palencia PF, Dueñas MT, Spano G, Fiocco D (2012) Beta-glucans improve growth, viability and colonization of probiotic microorganisms. Int $\mathrm{J}$ Mol Sci 13(5):6026-6039

Russo P, de Chiara MLV, Capozzi V, Arena MP, Amodio ML, Rascón A, Dueñas MT, López P, Spano G (2016) Lactobacillus plantarum strains for multifunctional oat-based foods. LWT Food Sci Technol 68:288-294

Ryan PM, Ross RP, Fitzgerald GF, Caplice NM, Stanton C (2015) Sugar-coated: exopolysaccharide producing lactic acid bacteria for food and human health applications. Food Funct 6(3): 679-693

Salehizadeh H, Shojaosadati SA (2001) Extracellular biopolymeric flocculants recent trends and biotechnological importance. Biotechnol Adv 19:371-385

Salehizadeh H, Vossoughi M, Alemzadeh I (2000) Some investigations on bioflocculant producing bacteria. Biochem Eng J 5(1):39-44

Sasikumar K, Vaikkath DK, Devendra L, Nampoothiri KM (2017) An exopolysaccharide (EPS) from a Lactobacillus plantarum BR2 with potential benefits for making functional foods. Bioresour Technol 241:1152-1156

Servin AL (2004) Antagonistic activities of lactobacilli and bifidobacteria against microbial pathogens. FEMS Microbiol $\operatorname{Rev} 28(4): 405-440$
Shen JW, Shi CW, Xu CP (2013) Exopolysaccharides from Pleurotus pulmonarius: fermentation optimization, characterization and antioxidant activity. Food Technol Biotechnol 51(4):520

Tsuda H, Miyamoto T (2010) Production of exopolysaccharide by Lactobacillus plantarum and the prebiotic activity of the exopolysaccharide. Food Sci Technol Res 16(1):87-92

Tsusaki K, Watanabe H, Nishimoto T, Yamamoto T, Kubota M, Chaen H, Fukuda S (2009) Structure of a novel highly branched $\alpha$-glucan enzymatically produced from maltodextrin. Carbohydr Res 344(16):2151-2156

Van Hijum SAFT, Szalowska E, Van Der Maarel MJEC, Dijkhuizen L (2004) Biochemical and molecular characterization of a levansucrase from Lactobacillus reuteri. Microbiology 150(3):621-630

Van Hijum SA, Kralj S, Ozimek LK, Dijkhuizen L, Van Geel-Schutten IG (2006) Structure-function relationships of glucansucrase and fructansucrase enzymes from lactic acid bacteria. Microbiol Mol Biol Rev 70(1):157-176

Wang Y, Ahmed Z, Feng W, Li C, Song S (2008) Physicochemical properties of exopolysaccharide produced by Lactobacillus kefiranofaciens ZW3 isolated from Tibet kefir. Int J Biol Macromol 43(3):283-288

Wang Y, Li C, Liu P, Ahmed Z, Xiao P, Bai X (2010) Physical characterization of exopolysaccharide produced by Lactobacillus plantarum KF5 isolated from Tibet Kefir. Carbohydr Polym 82(3):895-903

Wang K, Li W, Rui X, Chen X, Jiang M, Dong M (2014) Structural characterization and bioactivity of released exopolysaccharides from Lactobacillus plantarum 70810. Int J Biol Macromol 67:71-78

Wang J, Zhao X, Tian Z, Yang Y, Yang Z (2015a) Characterization of an exopolysaccharide produced by Lactobacillus plantarum YW11 isolated from Tibet kefir. Carbohydr Polym 125:16-25

Wang J, Zhao X, Yang Y, Zhao A, Yang Z (2015b) Characterization and bioactivities of an exopolysaccharide produced by Lactobacillus plantarum YW32. Int J Biol Macromol 74:119-126

Wang X, Shao C, Liu L, Guo X, Xu Y, Lü X (2017) Optimization, partial characterization and antioxidant activity of an exopolysaccharide from Lactobacillus plantarum KX041. Int J Biol Macromol 103: $1173-1184$

Wu Z, Lu J, Wang X, Hu B, Ye H, Fan J, Abid M, Zeng X (2014) Optimization for production of exopolysaccharides with antitumor activity in vitro from Paecilomyces hepiali. Carbohydr Polym 99: 226-234

Zannini E, Waters DM, Coffey A, Arendt EK (2016) Production, properties, and industrial food application of lactic acid bacteria-derived exopolysaccharides. Appl Microbiol Biotechnol 100(3):1121-1135

Zhang ZQ, Bo L, Xia SQ, Wang XJ, Yang AM (2007a) Production and application of a novel bioflocculant by multiple-microorganism consortia using brewery wastewater as carbon source. J Environ Sci 19(6):667-673

Zhang M, Cui SW, Cheung PCK, Wang Q (2007b) Antitumor polysaccharides from mushrooms: a review on their isolation process, structural characteristics and antitumor activity. Trends Food Sci Technol 18(1):4-19

Zhang L, Liu C, Li D, Zhao Y, Zhang X, Zeng X, Yang Z, Li S (2013) Antioxidant activity of an exopolysaccharide isolated from Lactobacillus plantarum C88. Int J Biol Macromol 54:270-275

Zhang Z, Liu Z, Tao X, Wei H (2016) Characterization and sulfated modification of an exopolysaccharide from Lactobacillus plantarum ZDY2013 and its biological activities. Carbohydr Polym 153:25-33

Zhu X, Zhao Y, Sun Y, Gu Q (2014) Purification and characterisation of plantaricin ZJ008, a novel bacteriocin against Staphylococcus spp. from Lactobacillus plantarum ZJ008. Food Chem 165:216-223

Zisu B, Shah NP (2005) Textural and functional changes in low-fat Mozzarella cheeses in relation to proteolysis and microstructure as influenced by the use of fat replacers, pre-acidification and EPS starter. Int Dairy J 15(6):957-972 